\title{
Genotype variant screening and phenotypic analysis of $F B N 1$ in Chinese patients with isolated ectopia lentis
}

\author{
YIJING ZHOU, DONGWEI GUO, QIANZHONG CAO, XINYU ZHANG, GUANGMING JIN and DANYING ZHENG
}

\author{
State Key Laboratory of Ophthalmology, Zhongshan Ophthalmic Center, \\ Sun Yat-sen University, Guangzhou, Guangdong 510060, P.R. China
}

Received June 18, 2020; Accepted January 5, 2021

DOI: $10.3892 / \mathrm{mmr} .2021 .11914$

\begin{abstract}
Isolated ectopia lentis (IEL) can lead to blindness as result of severe complications, such as retinal detachment and secondary glaucoma. Pathogenic variants in the fibrillin 1 (FBNI) gene are a common cause of IEL. The aim of the present study was to investigate the frequency of pathogenic FBN1 variants in twelve probands with IEL and to evaluate their associated phenotypes. Systemic clinical examination of the twelve probands indicated that all had bilateral EL with a median age at diagnosis of three years. High myopia was the most common feature among the probands (83.3\%; 10/12 cases). No extraocular symptoms (either cardiovascular or skeletal) were observed among these patients. Genomic DNA was extracted from peripheral blood leukocytes from all patients for targeted exome sequencing. Seven heterozygous missense variants in FBN1 were identified by bioinformatics analysis and further verified using Sanger sequencing. The seven variants were all classified as pathogenic after segregation analysis on available family members according to the American College of Medical Genetics and Genomics standards and guidelines. Of the seven variants, three were novel, namely c.2179T>C, c.2496T $>\mathrm{G}$ and c.3346G $>$ C. The remaining four, namely c. $184 \mathrm{C}>\mathrm{T}$, c. $367 \mathrm{~T}>\mathrm{C}$, c. $1879 \mathrm{C}>\mathrm{T}$ and c. $4096 \mathrm{G}>\mathrm{A}$ have been reported in previous studies. The seven pathogenic variants were identified in 8/12 (66.7\%) probands with IEL. These results expand the variant spectrum of the FBN1 gene as well as the understanding of the molecular pathogenesis of IEL.
\end{abstract}

\section{Introduction}

Congenital ectopia lentis (CEL) is characterized by displacement of the lens from its normal location (posterior to the iris

Correspondence to: Dr Danying Zheng or Dr Guangming Jin, State Key Laboratory of Ophthalmology, Zhongshan Ophthalmic Center, Sun Yat-sen University, 54 Xianlie South Road, Guangzhou, Guangdong 510060, P.R. China

E-mail: zhengdyy@163.com

E-mail: jingm@mail2.sysu.edu.cn

Key words: ectopia lentis, fibrillin-1, variant, Marfan syndrome and anterior to the vitreous body) as a result of congenital dysplasia of the suspensory ligament. It can lead to blindness due to complications, such as high myopia, irregular astigmatism, glaucoma, and retinal detachment $(1,2)$. The estimated prevalence rate of CEL was 6.4/100,000 in Denmark in $1998(3,4)$. CEL can occur in isolation; however, it is more common as a feature of disorders involving multiple systems (e.g., cardiovascular, skeletal, and nervous systems) and syndromic diseases, including Marfan syndrome [MFS; Online Mendelian Inheritance in Man (OMIM) no. 154700], homocystinuria (OMIM no. 236200), Weill-Marchesani syndrome (WMS; OMIM no. 277600), and Ehlers-Danlos syndrome (OMIM no. 225310) (5).

In a Danish study involving patients diagnosed with CEL, only $29.2 \%$ were diagnosed with isolated ectopia lentis (IEL) (3). Several studies have reported that CEL can not only be a monogenic disease but also an autosomal recessive inheritance (such as the ADAMTSL4 gene) (6-8). To our knowledge, IEL is most commonly caused by variants in the fillinbrin-1 (FBNI) gene. This gene is located on human chromosome $15 q 21$ and contains 66 exons spanning $235 \mathrm{~kb}(9,10)$. In 1994, Lönnqvist et al first described the p.Glu2447Lys FBN1 variant in a family with autosomal dominant EL inheritance and no cardiovascular disorders characteristic of MFS (11). To date, there have been reports of only 16 FBN1 variants causing IEL, of which 12 involved a cysteine substitution causing a missense variant and eight involved the EGF domain (12-18). Faivre et al (19) demonstrated that the cysteine substitution in the FBN1 protein was strongly associated with EL and that a missense variant in the 5'region of the FBN1 gene may be more likely to lead to EL. Yang et al (18) found that a cysteine insertion in the LTBP motif of the FBN1 protein could cause IEL. Liang et al (20) suggested that the p.Cys587Arg variant could affect the function of ciliary zonules, causing IEL; however, it may not affect the conformation of FBN1 or elastin fibers, nor cause any syndromic features.

Although $>2,500$ variants have been identified in FBN1, few have been associated with IEL (21). Variant spectrum and potential genotype-phenotype correlation analyses of the $F B N 1$ gene in patients with IEL would be valuable for understanding the mechanism of high phenotypic heterogeneity in this gene. Therefore, in this study, variant screening of FBN1 was carried out in twelve unrelated probands to investigate the genotype-phenotype association in patients with IEL. 


\section{Materials and methods}

Study participants. A total of twelve unrelated probands with IEL participated in this study. Detailed medical histories were obtained, and physical examinations were performed on each proband, including evaluation of the ophthalmic, cardiovascular, and skeletal systems. The ophthalmic examination included vision, intraocular pressure, dilated optometry results, slit lamp inspection, Pentacam, and Ultrasound Biomicroscope. In addition, a cardiac color Doppler ultrasound was performed to assess the aortic diameter and heart valve. Height, fingers and sternum were examined to evaluate the skeletal system. Patients were included if they had EL in one or both eyes and if their clinical features satisfied the 2010 Ghent criteria (22). Patients with a history of trauma (such as contusion of the eyeball) were excluded. This study was performed in accordance with The Declaration of Helsinki and was approved by The Institutional Review Board of the Zhongshan Ophthalmic Center, Sun Yat-sen University of Medicine. Written informed consent was obtained from all 12 participants (including the probands, their parents and the legal guardians of participants $<18$ years old) in accordance with The Declaration of Helsinki.

Variant detection. Genomic DNA was extracted from peripheral blood leucocytes of the 12 probands with IEL and their relatives using Maxwell 16 DNA Purification kit (Promega Corporation) according to the manufacturer's protocol. Targeted exome sequencing (TES) involving 126 genes associated with inherited eye diseases, including $F B N 1$, was performed on DNA samples from all probands, as described previously (23). Briefly, coding sequences of the 126 genes were captured using the SeqCap EZ Library SR V5 kit (cat. no. 7145594001; Roche NimbleGen, Inc.). A paired library was generated using a KAPA HTP Library Preparation kit (cat. no. 07961901001, Roche), then sequenced on the Illumina Miseq platform (Illumina, Inc.) using an Illumina Miseq v2 kit (cat. no. MS-102-2002, Roche; 300 cycles; paired-end). The average sequencing coverage was $\geq 125 x$. The raw sequencing data in fastq format were exported to the Strand NGS software (v2.6; Strand Life Sciences Pvt. Ltd.) to call single nucleotide variants and insertion-deletion variants. All small insertions, deletions indels and single-nucleotide variants (SNVs) were annotated and filtered using dbSNP138 InDels, NCBI dbSNP 146, NCBI RefSeq Gene (https://www. ncbi.nlm.nih.gov/refseq/rsg/), Human Genome Mutation Database (HGMD; Professional Version; Qiagen $\mathrm{GmbH}$ ), and 1000 Genomes Project, ExAC database (https://console.cloud. google.com/storage/browser/gnomad-public/legacy).

Pathogenic variants in FBN1 gene (GRCh37, accession number NC_000015.9). Variants in FBN1 were selected with a position ranging from 48700503 to 48937985 at chromosome 15 (Human GRCh37/hg19) from the TES data from the twelve probands. Common variants with an allelic frequency of $\geq 0.01$ according to the non-coding region or synonymous variations not affecting splicing were excluded. The pathogenicity of missense variants was predicted using several online tools, including Polyphen-2 (score 0-1, http://genetics.bwh.harvard. edu/pph2/), Combined Annotation Dependent Depletion (CADD; https://cadd.gs.washington.edu/), Rare Exome Variant Ensemble learner (REVEL score 0-1; https://sites. google.com/site/revelgenomics/), and ClinPred (score 0-1; release 2018.10; https://sites.google.com/site/clinpred/). The PhyloP software (https://ccg.epfl.ch//mga/hg19/phylop/phylop. html) and GERP++_RS software (http://mendel.stanford. edu/SidowLab/downloads/gerp/index.html) were used to analyze the conservation of variations. A missense variant would be classified as potentially pathogenic if it was predicted to be deleterious by at least two of the four prediction tools. The sequences of potential pathogenic variants were validated using Sanger sequencing. Protein structures were predicted using wild-type and mutant amino acid sequences using Swiss-model software (https://swissmodel.expasy.org/). All potential variants were classified manually according to the guidelines of the American College of Medical Genetics and Genomics (24). Comparison between the results of the present study with the main available variants in FBN1 reported by previous literatures was also performed $(25,26)$.

Statistical analysis. The differences in the genomic position of the $F B N 1$ variants and in the age at diagnosis between patients with MFS or IEL were analyzed using Mann-Whitney U test. Data analysis was performed using the SPSS statistical package (version 26.0 for Windows; IBM Corp.) $\mathrm{P}<0.05$ was considered to indicate a statistically significant difference.

\section{Results}

Clinical findings. A total of twelve probands participated in the study, including nine male and three female patients. Clinical information of all patients is displayed in Table I. The age at examination ranged from 3 to 27 years old, with a mean age of diagnosis of 9.91 years $( \pm 8.85$ years). All subjects had symptoms of poor vision and were diagnosed with IEL. Eleven were first diagnosed with IEL before six years of age, while the remaining proband was first diagnosed at 20 years of age. All patients with IEL in the present study presented lens subluxation or luxation in both eyes and none showed extraocular (cardiovascular or skeletal) features. High myopia was a common feature of these probands (10/12 cases; 83.33\%). The other two patients had hyperopia. Except for probands 4 and 9 , who were not eligible for surgery due to their young age, all other probands underwent transscleral-fixated intraocular lens implantation, which effectively improved their vision.

Variants in FBN1 gene. In total, seven missense variants in FBN1 were identified in eight of the 12 probands with EL, and no variant was detected in the other four probands. The variant frequency was $66.67 \%$. All eight variants of $F B N 1$ were de novo, since they were not detected in the parents of the eight probands. The pedigrees of eight families are displayed in Fig. 1. In total, three (c.2179T>C, c.2496T $>$ G and c.3346G $>C$ ) of the eight variants were novel variants. The other four (c.184C $>$ T, c.367T $>$ C, c. 1879C $>$ T and c.4096G $>$ A) had been reported previously (Table II; Fig. 2) (27-29). All eight variants were located at the calcium-binding epithermal growth factor (cbEGF)-like domain (Fig. 3) and were predicted to be deleterious by all four prediction tools (Table II).

The lens dislocation of a patient with a heterozygous $\mathrm{c}$. 367T $>\mathrm{C}$ (p.Cys123Arg) variant in FBN1 is shown in Fig. 4. 
Table I. Characteristics of patients with IEL in the present study.

\begin{tabular}{|c|c|c|c|c|c|c|c|c|c|c|}
\hline \multirow[b]{2}{*}{ Patient } & \multirow[b]{2}{*}{$\begin{array}{l}\text { Age, } \\
\text { years }\end{array}$} & \multirow[b]{2}{*}{ Sex } & \multirow[b]{2}{*}{$\begin{array}{l}\text { Age at, } \\
\text { diagnosis } \\
\text { years }\end{array}$} & \multicolumn{4}{|c|}{ Ocular examination } & \multicolumn{3}{|c|}{ Extraocular system } \\
\hline & & & & $\begin{array}{c}\text { VA, } \\
(\log \mathrm{MAR}) \\
\mathrm{OD} / \mathrm{OS}\end{array}$ & $\begin{array}{l}\text { Preoperative } \\
\text { Refraction, } \\
\text { OD/OS (D) }\end{array}$ & $\begin{array}{l}\text { Surgery } \\
\text { for IEL }\end{array}$ & $\begin{array}{c}\text { Postoperative } \\
\text { BCVA, } \\
\text { (logMAR) } \\
\text { OD/OS }\end{array}$ & $\begin{array}{l}\text { Skeletal } \\
\text { anomaly }\end{array}$ & $\begin{array}{c}\mathrm{AO} \\
\text { diameter, } \\
\mathrm{mm}\end{array}$ & $\begin{array}{c}\text { Cardiovascular } \\
\text { symptom }\end{array}$ \\
\hline 1 & 6 & M & 2 & $0.5 / 1.3$ & $-13.5 /-9.5$ & Yes & $0.09 / 0.3$ & None & 22 & None \\
\hline 2 & 5 & M & 4 & $1.3 / 1.0$ & $+14.0 /+14.75$ & Yes & $0.3 / 0.0$ & None & 18 & None \\
\hline 3 & 4 & M & 3 & $1.2 / 1.0$ & $-10.0 /-12.0$ & Yes & $0.15 / 0.0$ & None & 23 & None \\
\hline 4 & 3 & M & 3 & NA & $-7.75 /-8.0$ & No & NA & None & 18 & None \\
\hline 5 & 3 & M & 3 & $1.3 / 1.5$ & $-24.0 /-23.0$ & Yes & $0.15 / 0.3$ & None & 23 & None \\
\hline 6 & 4 & M & 3 & $1.3 / 0.6$ & $-14.0 /-18.0$ & Yes & $0.15 / 0.3$ & None & 17 & None \\
\hline 7 & 11 & $\mathrm{~F}$ & 5 & $1.3 / 1.5$ & $-8.0 /-7.0$ & Yes & $0.15 / 0.09$ & None & 29 & None \\
\hline 8 & 27 & $\mathrm{~F}$ & 5 & $0.7 / 0.9$ & $-10.0 /-14.0$ & Yes & $0.04 / 0.00$ & None & 29 & None \\
\hline 9 & 4 & M & 3 & $0.6 / 1.0$ & $+2 /+2$ & No & NA & None & 18 & None \\
\hline 10 & 26 & $\mathrm{~F}$ & 6 & $2.0 / 1.3$ & $-18.0 /-14.0$ & Yes & NA/0.00 & None & 27 & None \\
\hline 11 & 21 & M & 20 & $0.3 / 1.3$ & $-17.0 /-8.5$ & Yes & $0.15 / 0.00$ & None & NA & None \\
\hline 12 & 5 & $\mathrm{M}$ & 3 & $1.1 / 0.9$ & $-7.5 /-5.5$ & Yes & $0.15 / 0.00$ & None & 16 & None \\
\hline
\end{tabular}

VA, visual acuity; OD, oculus dexter; OS, oculus sinister; D, degree; BCVA, best corrected visual acuity; logMAR, logarithm of the minimum angle of resolution; NA, not available; IEL, isolated ectopia lentis; AO, aortic.

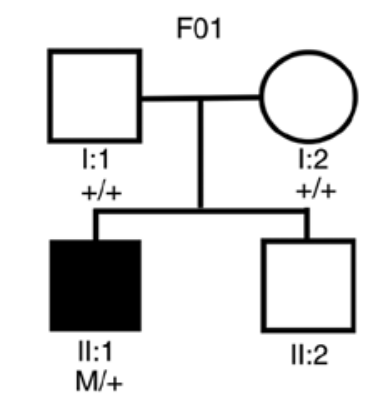

M:c. $184 \mathrm{C}>\mathrm{T}$
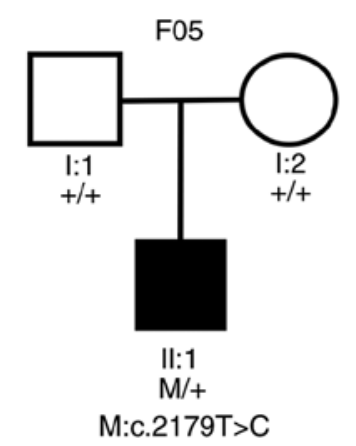
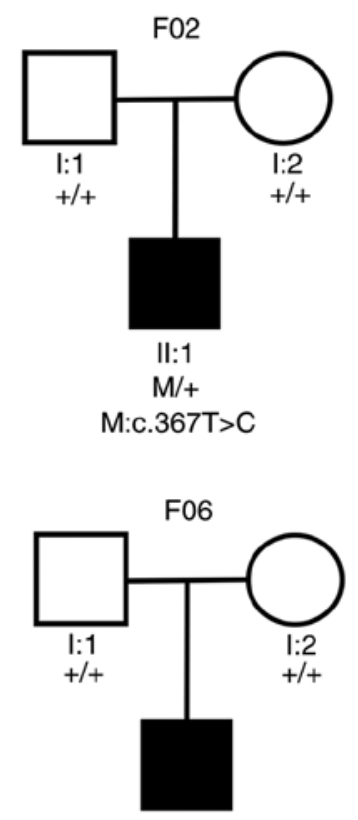

II:1

$\mathrm{M} /+$

M:c.2496T>G

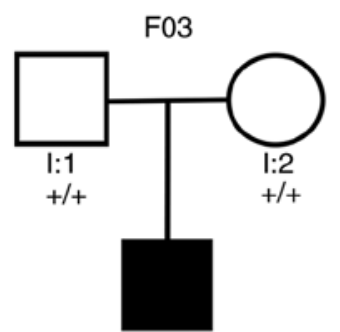

II:1

$\mathrm{M} /+$

M:c. $1879 \mathrm{C}>\mathrm{T}$

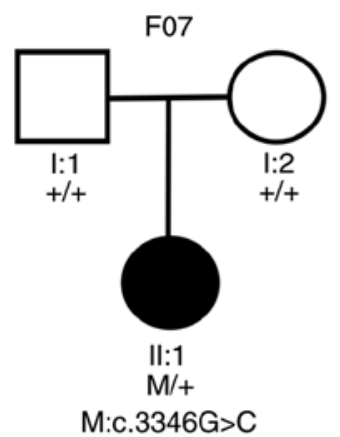

F04
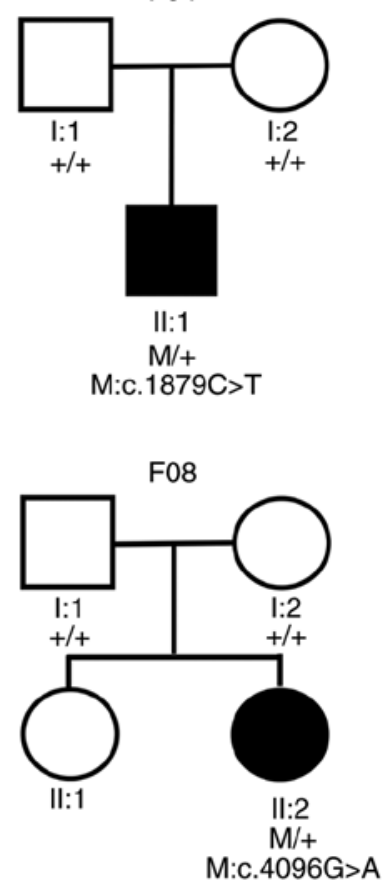

Figure 1. Pedigrees of eight families with fibrillin-1 variants. The filled squares (male) and circles (female) are the affected individuals. The + symbol indicates carriers of the wild-type gene. I and II indicate the generation. F, family; M, mutant.

The main reported variants in FBN1 gene were collected and shown in Table SI, the average age of patients is 24.6 years old, and there is no obvious trend in the mutation sites of the FBN1 gene. Compared with patients with MFS, patients with IEL were diagnosed at younger ages (Fig. 5A; $\mathrm{P}<0.001$ ). Moreover, the variants identified in the probands with IEL in the present study were located in upstream coding regions of the gene (from exon 2 to exon 33; Table II), whereas variants associated with MFS were located throughout the whole region of the gene as reported by previous studies $(25,26)$. The genomic positions of the FBN1 gene variants were in the upstream regions of chromosome $15(\mathrm{P}=0.04$; Fig. 5B). 


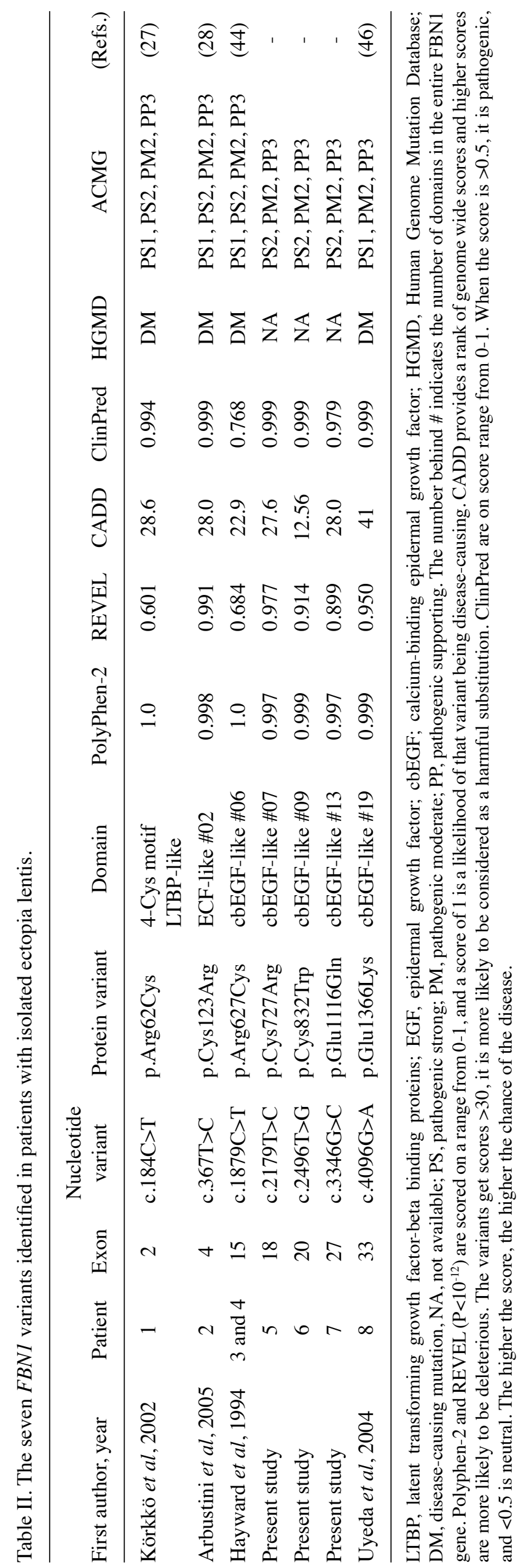

\section{Discussion}

CEL is an autosomal dominant connective tissue disorder. Several FBN1 variants have been reported to be pathogenic for CEL, the majority of which are associated with syndromic CEL (including MFS) (19). However, only a few studies have examined $F B N 1$ variants in patients with IEL (30). In the present study, seven $F B N 1$ variants in eight patients with IEL, including three novel variants and four previously reported variants.

The FBN1 gene encodes the fibrillin 1 protein, which is an essential element of microfibrils found in elastic and nonelastic tissues, including ciliary bodies, blood vessels, lungs, and skin (5). In patients with MFS, WMS or IEL (31), >2,500 variants have been identified in this gene, most of which were identified in patients with MFS. It has been reported that variants in $F B N 1$ accounted for $\sim 90 \%$ of patients with MFS (32). However, only 16 identified variants in the $F B N 1$ are associated with IEL (30), consistent with the variant frequency in the present study. Missense variants account for two-thirds of the 2,500 known variants in FBNI (19). Most missense variants are located at the cbEGF domain, and $\sim 70 \%$ of these are conserved cysteine substitutions (33). Cysteine plays an important role in the EGF-like domain of the FBN1 protein. Indeed, substitutions of cysteine residues can disrupt one of three disulfide bridges that covalently connect three pairs of cysteine residues that are highly conserved in EGF-like domains, thereby affecting the structural stability of the domain itself and neighboring domains as well $(34,35)$. Previous studies have suggested that missense variants in FBNI are associated with EL phenotype $(19,36)$. EL is more likely when the missense variants involve cysteine residues (37). In the present study, missense variants were detected in eight probands, of which six were located in the cbEGF domain, and five involved a cysteine residue. The present findings are consistent with those of the previous study in which EL was associated with missense mutations and cysteine residue change (37).

A total of three novel heterozygous variants (c.2179T $>\mathrm{C} / \mathrm{p}$. Cys727Arg; c.2496T >G/p. Cys832Trp; c. 3346G >C/p. Glu1116Gln) in $F B N 1$ were identified in three probands with IEL. The initial symptoms experienced by the three probands included blurred vision. Only high myopia and EL were present in these three probands without extraocular features. All three novel variants are de novo and were predicted to have a possible pathogenic effect according to PolyPhen-2 and SIFT. In addition, the two novel variants, p.Cys727Arg and p.Cys832Trp, occurred at the same positions as the known p.Cys727Tyr and p.Cys832Tyr respectively. These two variants may abolish a disulfide bond and affect the first (C1) and sixth (C6) conserved cysteine of the cbEGF domain that are essential for correct EGF-like domain structure $(38,39)$. The variant c. $3346 \mathrm{G}>\mathrm{C}$ is located in cbEGF domain and changes the conserved glutamic acid residue. It is suggested that the change of glutamic acid may affect $\mathrm{Ca}^{2+}$ binding of cbEGF domain and destructs the structure of FBN1 gene (35).

The variant c. $184 \mathrm{C}>\mathrm{T}$ carried by proband 1 has previously been associated with late-onset IEL $(38,40)$. In a Chinese family, the age of diagnosis ranged between 41 and 65 years (41). In contrast with these previous findings, in the present study, the patient with this variant developed EL symptoms at age 3, however in this study it was found that it could develop at 2 years old. The c.367T $>\mathrm{C}$ variant is associated with classical MSF, 


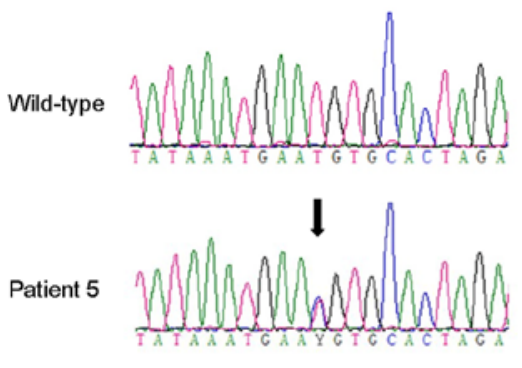

FBN1 c. 2179T $>$ C
Wild-type

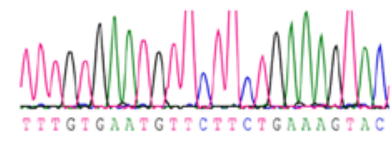

Patient 6

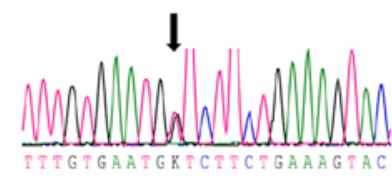

FBN1 c.2496T>G

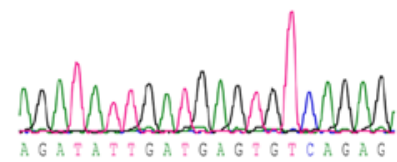

Patient 7

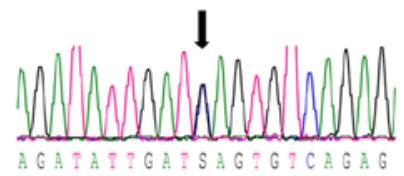

FBN1 c.3346G>C

Figure 2. Fragment of $F B N 1$ cDNA sequence in probands 5, 6 and 7. Sequencing traces from probands 5,6 and 7 with the c.2179T $>C$, c.2496T $>\mathrm{G}$ and c.3346G $>$ C variants, respectively. $F B N 1$, fibrillin 1.

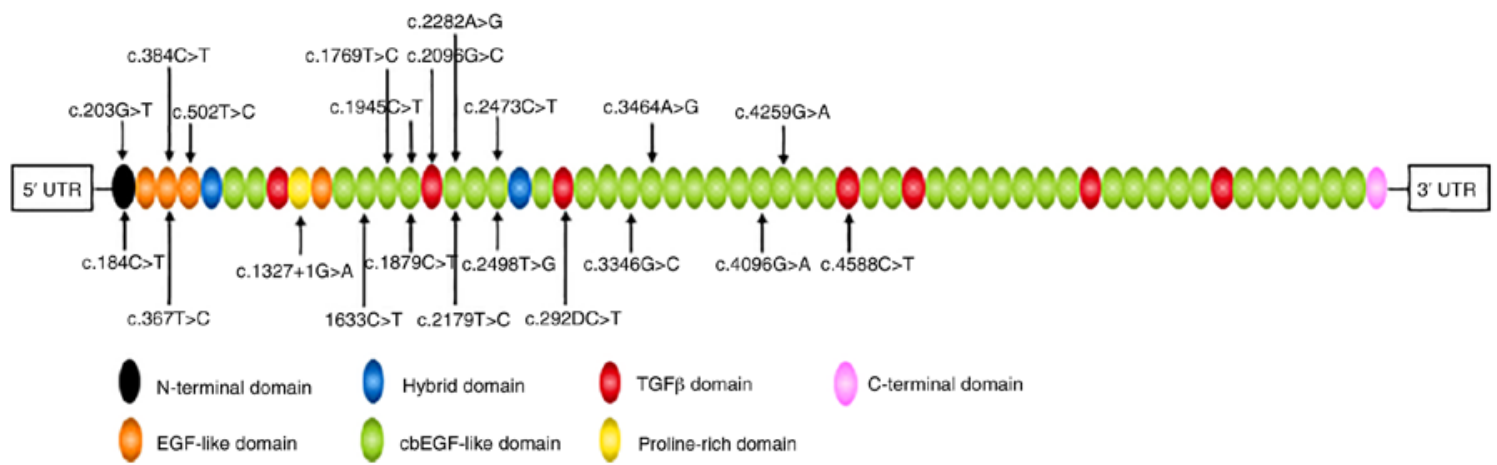

Figure 3. Schematic presentation of $F B N 1$ variants. The position of reported $F B N 1$ variants associated with isolated ectopia lentis from the present study and the literature (29). FBN1, fibrillin 1; EGF, epidermal growth factor; cbEGF, calcium-binding epidermal growth factor; UTR, untranslated region.

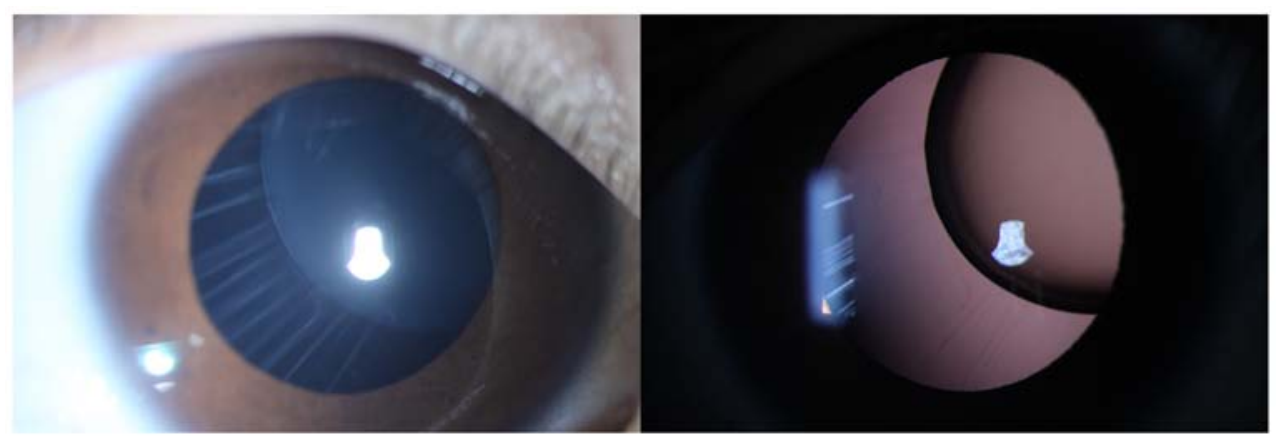

Figure 4. The Anterior segment photography of proband 2 with a heterozygous c. 367T $>C$ (p.Cys123Arg) fibrillin 1 variant. The left panel shows a clear dislocation of the lens, while the right panel shows the elongated lens zonule using slit lamp photography.
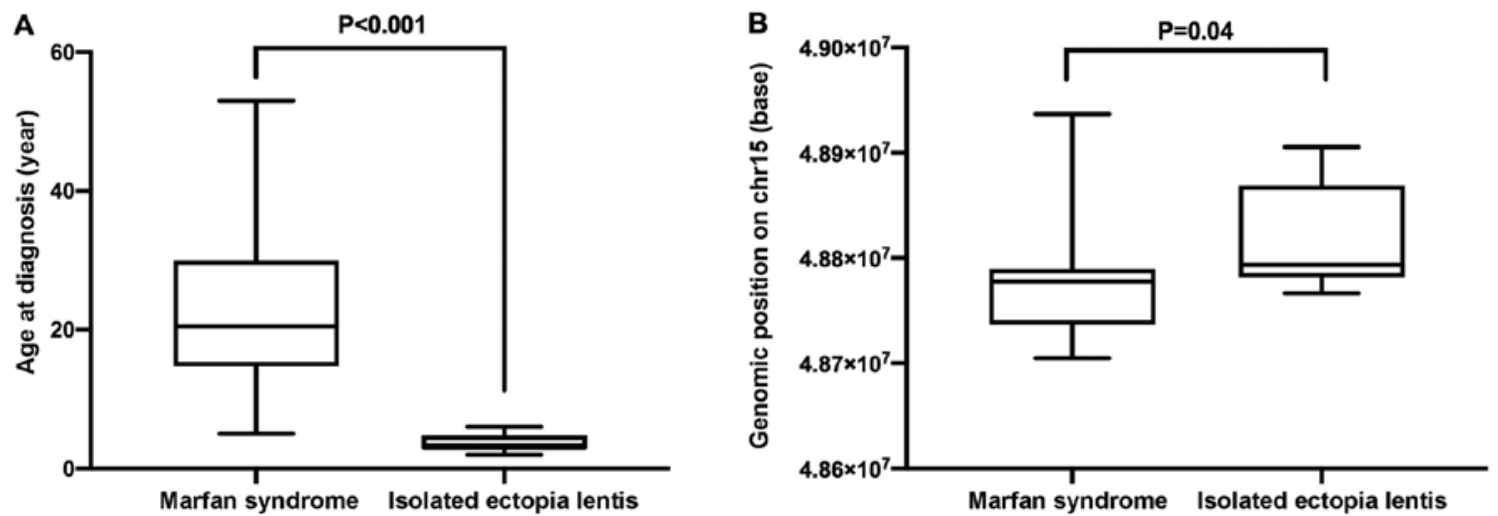

Figure 5. The mutation distributions from different diagnosis ages of MFS and IES patients. Comparison of age at diagnosis (A) and genomic position of the fibrillin 1 variants (B) in patients with Marfan syndrome or isolated ectopia lentis. chr15, chromosome 15. 
was identified in a Frenchman in 2005 (28). The phenotype of the patients in this study only involved the ocular system. The c.1879C $>$ T variant was reported in 1994 and causes manifestations in the ocular and skeletal systems, with mild aortic involvement (29), but the present study did not find any system disorder in probands. The c. $4096 \mathrm{G}>\mathrm{A}$ variant was identified in 2004 in two patients, one with cardiovascular manifestations but no ocular signs, and the other with MFS (36) and no cardiovascular system signs were found in any of the probands in the present study.

Mutations in the FBNl gene are commonly associated with MFS. However, in the present study, the eight probands with FBN1 variants, did not have any systemic disorders except for IEL. The examination of aortic diameter indicated that these patients did not meet the standards for abnormal aortic roots, nor did they meet the revised Ghent criteria for MFS (22). Nevertheless, it remains possible that the patients could develop aortic root dilation or other manifestations of MFS in the future. The present findings also suggested that ocular disorders might occur in isolation or may be the first sign of the syndrome. Currently, it remains difficult to diagnose MFS in young patients with EL; as a result, these pediatric patients still require long-term annual cardiovascular imaging.

In the present study, the $F B N 1$ variant identified in the eight probands were located in the first 33 exons, and some involved arginine-to-cysteine substitutions. Faivre et al (19) also noted that EL was more likely when missense changes occurred in the exon 1-21. Compared with patients with MFS, the average genomic positions of the variants carried by the eight probands with IEL were located more upstream of the FBN1 gene. Moreover, IEL was diagnosed at a younger age, compared with MFS. This result is consistent with those of previous studies describing EL as the first symptom of MFS, occurring before the age of 10 (42). Most probands with FBNl gene variant suffered from poor visual acuity, significant EL, and high myopia. Most also underwent surgical treatment, except for proband 11 who was older than 20 years, and proband 4 , who was not eligible for surgery due to parents' disagreement. Therefore, it may be hypothesized that these variants lead to earlier ocular symptoms.

The limitations of the current study were that the patients were young; patients may not have developed other clinical manifestations at the time of the present study. In addition to MFS and EL, the FBNI gene has also been implicated in conditions such as WMS, isolated ascending aorta, mitral valve prolapse, aortic root dilatation without dissection (43), and familial arachnodactyly (44). Associations between genotype and phenotype have not been determined. In addition to the FBN1 gene, IEL can also be inherited in an autosomal recessive fashion because of ADAMTS4, identified in 2009; variants in this gene can cause earlier ocular phenotypes than those associated with $F B N 1$ in Caucasian populations $(16,45)$. Altogether, the evidence suggests a need for the identification of other target genes and examination of larger patient cohorts in future studies. Further study is required to examine associations between genotype and phenotype in the FBN1 gene. Early genetic screening contributes to early disease prediction and management for patients and their families.

In summary, three novel variants and four reported variants were identified in the FBN1 gene from eight Chinese probands with IEL. These findings further enrich the FBN1 variant spectrum and may provide insight into the clinical diagnosis and management of IEL.

\section{Acknowledgements}

Not applicable.

\section{Funding}

This work was supported by The National Natural Science Foundation of China (grant nos. 81873673 and 81900841) and The Fundamental Research Funds of the State Key Laboratory of Ophthalmology (grant no. 30306020240020212); Dr Guangming Jin receives support from The Young Teachers Training Program of Sun Yat-sen University (grant no. 20ykpy143).

\section{Availability of data and materials}

The datasets used and/or analyzed during the current study are available from the corresponding author on reasonable request. The sequencing datasets are also available from the Sequence Read Archive under BioProject no. PRJNA668437.

\section{Authors' contributions}

YZ, GJ and DZ conceived and designed the experiments. YZ performed the experiments. DG, QAC and XZ analyzed the data. YZ, DG, QAC and XZ contributed reagents/materials/analysis tools. YZ, GJ and DG wrote the manuscript. GJ and DZ confirmed the authenticity of the data in the present study. All authors read and approved the final manuscript.

\section{Ethics approval and consent to participate}

This study was performed in accordance with The Declaration of Helsinki and was approved by The Institutional Review Board at the Zhongshan Ophthalmic Center, Sun Yat-sen University of Medicine. Written informed consent was obtained from all participants and from the legal guardians of participants $<18$ years old, in accordance with The Declaration of Helsinki.

\section{Patient consent for publication}

All participants provided informed consent (including the probands, their parents and the legal guardians of participants under 18 years old) for the publication of their data, including images and examination results.

\section{Competing interests}

The authors declare that they have no competing interests.

\section{References}

1. Maumenee IH: The eye in the marfan syndrome. Trans Am Ophthalmol Soc 79: 684-733, 1981.

2. Chandra A and Charteris D: Molecular pathogenesis and management strategies of ectopia lentis. Eye (Lond) 28: 162-168, 2014.

3. Fuchs J and Rosenberg T: Congenital ectopia lentis. A Danish national survey. Acta Ophthalmol Scand 76: 20-26, 1998.

4. Sadiq MA and Vanderveen D: Genetics of ectopia lentis. Semin Ophthalmol 28: 313-320, 2013. 
5. Nelson LB and Maumenee IH: Ectopia lentis. Surv Ophthalmol 27 143-160, 1982.

6. Ruiz C, Rivas F, Villar-Calvo VM, Serrano-Lucas JI and Cantú JM: Familial simple ectopia lentis. A probable autosomal recessive form. Ophthalmic Paediatr Genet 7: 81-84, 1986.

7. Sinha A and Rahman A: Ectopia lentis in a family. Indian J Ophthalmol 28: 33-35, 1980.

8. Casper DS, Simon JW, Nelson LB, Porter IH and Lichtenstein SB: Familial simple ectopia lentis: A case study. J Pediatr Ophthalmol Strabismus 22: 227-230, 1985.

9. Sakai LY, Keene DR and Engvall E: Fibrillin, a new 350-kD glycoprotein, is a component of extracellular microfibrils. J Cell Biol 103 2499-2509, 1986

10. Tynan K, Comeau K, Pearson M, Wilgenbus P, Levitt D, Gasner C, Berg MA, Miller DC and Francke U: Mutation screening of complete fibrillin-1 coding sequence: Report of five new mutations, including two in 8-cysteine domains. Hum Mol Genet 2: 1813-1821, 1993.

11. Lönnqvist L, Child A, Kainulainen K, Davidson R, Puhakka L and Peltonen L: A novel mutation of the fibrillin gene causing ectopia lentis. Genomics 19: 573-576, 1994

12. Jin C, Yao K, Jiang J, Tang X, Shentu X and Wu R: Novel FBN1 mutations associated with predominant ectopia lentis and marfanoid habitus in Chinese patients. Mol Vis 13: 1280-1284, 2007.

13. Deng T, Dong B, Zhang X, Dai H and Li Y: Late-Onset bilateral lens dislocation and glaucoma associated with a novel mutation in FBN1. Mol Vis 14: 1229-1233, 2008.

14. Turner CL, Emery H, Collins AL, Howarth RJ, Yearwood CM, Cross E, Duncan PJ, Bunyan DJ, Harvey JF and Foulds NC: Detection of 53 FBN1 mutations (41 novel and 12 recurrent) and genotype-phenotype correlations in 113 unrelated probands referred with Marfan syndrome, or a related fibrillinopathy. Am J Med Genet A 149A: 161-170, 2009.

15. Zadeh N, Bernstein JA, Niemi AK, Dugan S, Kwan A, Liang D, Hyland JC, Hoyme HE, Hudgins L and Manning MA: Ectopia lentis as the presenting and primary feature in marfan syndrome. Am J Med Genet A 155A: 2661-2668, 2011

16. Chandra A, Aragon-Martin JA, Hughes K, Gati S, Reddy MA Deshpande C, Cormack G, Child AH, Charteris DG and Arno G: A genotype-phenotype comparison of ADAMTSL4 and FBN1 in isolated ectopia lentis. Invest Ophthalmol Vis Sci 53: 4889-4896, 2012.

17. Li H, Qu W, Meng B, Zhang S, Yang T, Huang S and Yuan H: Identification and study of a FBN1 gene mutation in a Chinese family with ectopia lentis. Mol Vis 18: 504-511, 2012.

18. Yang G, Chu M, Zhai X and Zhao J: A novel FBN1 mutation in a Chinese family with isolated ectopia lentis. Mol Vis 18: 945-950, 2012.

19. Faivre L, Collod-Beroud G, Loeys BL, Child A, Binquet C, Gautier E, Callewaert B, Arbustini E, Mayer K et al: Effect of mutation type and location on clinical outcome in 1,013 probands with Marfan syndrome or related phenotypes and FBN1 mutations: an international study. Am J Hum Genet 81: 454-466, 2007.

20. Liang C, Fan W, Wu S and Liu Y: Identification of a novel FBN1 mutation in a Chinese family with isolated ectopia lentis. Mol Vis 17: 3481-3485, 2011

21. Yang H, Ma Y, Luo M, Zhao K, Zhang Y, Zhu G, Sun X, Luo F, Wang L et al: Identification of gross deletions in FBN1 gene by MLPA. Hum Genomics 12: 46, 2018.

22. Loeys BL, Dietz HC, Braverman AC, Callewaert BL, De Backer J, Devereux RB, Hilhorst-Hofstee Y, Jondeau G, Faivre L et al: The revised Ghent nosology for the Marfan syndrome. J Med Genet 47: 476-485, 2010

23. Wang P, Li S, Sun W, Xiao X, Jia X, Liu M, Xu L, Long Y and Zhang Q: An ophthalmic targeted exome sequencing panel as a powerful tool to identify causative mutations in patients suspected of hereditary eye diseases. Transl Vis Sci Technol 8: 21, 2019.

24. Richards S, Aziz N, Bale S, Bick D, Das S, Gastier-Foster J, Grody WW, Hegde M, Lyon E, Spector E, et al: Standards and guidelines for the interpretation of sequence variants: A joint consensus recommendation of the American college of medical genetics and genomics and the association for molecular pathology. Genet Med 17: 405-424, 2015.

25. Chung BH, Lam ST, Tong TM, Li SY, Lun KS, Chan DH, Fok SF Or JS, Smith DK, Yang W and Lau YL: Identification of novel FBN1 and TGFBR 2 mutations in 65 probands with marfan syndrome or marfan-like phenotypes. Am J Med Genet A 149A: 1452-1459, 2009

26. Yang H, Luo M, Chen Q, Fu Y, Zhang J, Qian X, Sun X, Fan Y, Zhou Z and Chang Q: Genetic testing of the FBN1 gene in Chinese patients with marfan/marfan-like syndrome. Clin Chim Acta 459: $30-35,2016$.
27. Körkkö J, Kaitila I, Körkkö L, Peltonen L and Ala-Kokko L: Sensitivity of conformation sensitive gel electrophoresis in detecting mutations in marfan syndrome and related conditions. J Med Genet 39: 34-41, 2002.

28. Arbustini E, Grasso M, Ansaldi S, Malattia C, Pilotto A, Porcu E, Disabella E, Marziliano N,Pisani A, Lanzarini L, et al: Identification of sixty-two novel and twelve known FBN1 mutations in eighty-one unrelated probands with marfan syndrome and other fibrillinopathies. Hum Mutat 26: 494, 2005.

29. Hayward C, Rae AL, Porteous ME, Logie LJ and Brock DJ: Two novel mutations and a neutral polymorphism in EGF-like domains of the fibrillin gene (FBN1): SSCP screening of exons 15-21 in Marfan syndrome patients. Hum Mol Genet 3: 373-375, 1994.

30. Zhang L, Lai YH, Capasso JE, Han S and Levin AV: Early onset ectopia lentis due to a FBN1 mutation with non-penetrance. Am J Med Genet A 167: 1365-1368, 2015.

31. Sakai LY, Keene DR, Renard M and De Backer J: FBN1: The disease-causing gene for marfan syndrome and other genetic disorders. Gene 591: 279-291, 2016

32. Baetens M, Van Laer L, De Leeneer K, Hellemans J, De Schrijver J, Van De Voorde H, Renard M, Dietz H, Lacro RV, Menten B, et al: Applying massive parallel sequencing to molecular diagnosis of marfan and loeys-dietz syndromes. Hum Mutat 32: 1053-1062, 2011.

33. Seo GH, Kim YM, Kang E, Kim GH, Seo EJ, Lee BH, Choi JH and Yoo HW: The phenotypic heterogeneity of patients with Marfan-related disorders and their variant spectrums. Medicine (Baltimore) 97: e10767, 2018.

34. Aoyama T, Tynan K, Dietz HC, Francke U and Furthmayr H: Missense mutations impair intracellular processing of fibrillin and microfibril assembly in marfan syndrome. Hum Mol Genet 2: 2135-2140, 1993.

35. Downing AK, Knott V, Werner JM, Cardy CM, Campbell ID and Handford PA: Solution structure of a pair of calcium-binding epidermal growth factor-like domains: Implications for the marfan syndrome and other genetic disorders. Cell 85: 597-605, 1996.

36. Comeglio P, Johnson P, Arno G, Brice G, Evans A, Aragon-Martin J, da Silva FP, Kiotsekoglou A and Child A: The importance of mutation detection in marfan syndrome and marfan-related disorders: Report of 193 FBN1 mutations. Hum Mutat 28: 928, 2007.

37. Schrijver I, Liu W, Brenn T, Furthmayr H and Francke U: Cysteine substitutions in epidermal growth factor-like domains of fibrillin-1: Distinct effects on biochemical and clinical phenotypes. Am J Hum Genet 65: 1007-1020, 1999.

38. Stheneur C, Collod- Béroud G, Faivre L, Buyck JF, Gouya L, Le Parc JM, Moura B, Muti C, Grandchamp B, Sultan G, et al: Identification of the minimal combination of clinical features in probands for efficient mutation detection in the FBN1 gene. Eur J Hum Genet 17: 1121-1128, 2009.

39. Liu WO, Oefner PJ, Qian C, Odom RS and Francke U: Denaturing HPLC-identified novel FBN1 mutations, polymorphisms, and sequence variants in marfan syndrome and related connective tissue disorders. Genet Test 1: 237-242, 1997.

40. Zhao JH, Jin TB, Liu QB, Chen C and Hu HT: Ophthalmic findings in a family with early-onset isolated ectopia lentis and the p.Arg62Cys mutation of the fibrillin-1 gene (FBN1). Ophthalmic Genet 34: 21-26, 2013.

41. Yu R, Lai Z,Zhou W, Ti DD and Zhang XN: Recurrent FBN1 mutation (R62C) in a Chinese family with isolated ectopia lentis. Am J Ophthalmol 141: 1136-1138, 2006.

42. Tinkle BT, Saal HM and Committee on Genetics: Health supervision for children with marfan syndrome. Pediatrics 132: e1059-e1072, 2013.

43. Milewicz DM, Michael K, Fisher N, Coselli JS, Markello T and Biddinger A: Fibrillin-1 (FBN1) mutations in patients with thoracic aortic aneurysms. Circulation 94: 2708-2711, 1996.

44. Hayward C, Porteous ME and Brock DJ: A novel mutation in the fibrillin gene (FBN1) in familial arachnodactyly. Mol Cell Probes 8: 325-327, 1994.

45. Ahram D, Sato TS, Kohilan A, Tayeh M, Chen S, Leal S, Al-Salem $M$ and El-Shanti H: A homozygous mutation in ADAMTSL4 causes autosomal-recessive isolated ectopia lentis. Am J Hum Genet 84: 274-278, 2009.

46. Uyeda T, Takahashi T, Eto S, Sato T, Xu G, Kanezaki R, Toki T, Yonesaka S and Ito E: Three novel mutations of the fibrillin-1 gene and ten single nucleotide polymorphisms of the fibrillin-3 gene in marfan syndrome patients. J Hum Genet 49: 404-407, 2004.

This work is licensed under a Creative Commons Attribution-NonCommercial-NoDerivatives 4.0 International (CC BY-NC-ND 4.0) License. 